\section{Mechanisms of thrombosis in antiphospholipid syndrome}

One of the characteristics of antiphospholipid syndrome (APS) is an increased risk of thrombotic complications. Antiphospholipid antibodies (present in the serum of patients with APS) are known to induce platelet activation and the production of procoagulants such as von Willebrand factor, thereby encouraging thrombosis; however, the exact mechanism is unknown. Whereas most previous studies were conducted under static conditions, Levy et al. used a cone-and-plate(let) analyzer to subject blood samples on an adhesive surface to shear stress, allowing assessment of platelet adhesion and aggregation under conditions closer to those that occur in vivo.

The blood donors included 88 hospitalized patients with APS, with or without a history of thrombosis; 17 patients without antiphospholipid antibodies but with a history of thrombosis; and 26 healthy controls. APS patients with a history of thrombosis had increased platelet adhesion and aggregation compared with patients with no history of thrombosis and controls, who both showed normal results $(P<0.01)$. Preincubation with the recombinant von Willebrand factor fragment RG12986 inhibited platelet adhesion and aggregation, in both a subgroup of APS patients with thrombotic events and controls. No relationship was found between thrombosis in patients with APS and type of antiphospholipid antibody.

The authors suggest that at there are at least two mechanisms of platelet activation in APS: platelet activation by antiphospholipid antibodies and high platelet deposition under shear stress induced by von Willebrand factor.

Katherine Sole

Original article Levy Y et al. (2005) Increased platelet deposition on extracellular matrix under flow conditions in patients with antiphospholipid syndrome who experience thrombotic events. Arthritis Rheum 52: 4011-4017

\section{Gastrointestinal safety of COX2 inhibitors might be no better than that of nonselective NSAIDs}

Cyclo-oxygenase 2 (COX2) inhibitors were developed to provide pain relief without the gastrointestinal side effects associated with nonsteroidal anti-inflammatory drugs (NSAIDs); however, trial data that support the hypothetical gastrointestinal safety of COX2 inhibitors are sparse. A UK population-based nested casecontrol study that compared the risk of upper gastrointestinal events in patients receiving either COX2 inhibitors or nonselective NSAIDs casts further doubt on this hypothesis; no consistent evidence was found that new COX2 inhibitors have better gastrointestinal safety than nonselective NSAIDs.

Participants were identified from the QRESEARCH database, which includes medical records of over 7 million UK patients. The investigators compared 9,407 case patients aged over 25, who had their first adverse upper gastrointestinal event between August 2000 and July 2004 , with 88,867 matched controls, who had no gastrointestinal events.

Among the case patients, $45.2 \%$ had been prescribed a nonselective NSAID and $9.9 \%$ had been prescribed a COX2 inhibitor, compared with $33.3 \%$ and $5.6 \%$ of controls, respectively. After adjusting for confounding variables, there was an increased risk of gastrointestinal events with current use of naproxen, diclofenac, other COX2 inhibitors, other nonselective NSAIDs, aspirin, rofecoxib and ibuprofen. There was no increased risk with current use of celecoxib, but patient numbers were low. Patients prescribed ulcer-healing drugs plus an NSAID did not have an elevated risk of gastrointestinal events, except for those prescribed diclofenac plus an ulcer-healing drug; these patients had a small increased risk compared with controls.

Rachel Murphy

Original article Hippisley-Cox J et al. (2005) Risk of adverse gastrointestinal outcomes in patients taking cyclo-oxygenase-2 inhibitors or conventional non-steroidal anti-inflammatory drugs: population based nested case-control analysis. BMJ 331: $1310-1316$

\section{Serum biomarkers predict joint damage in rheumatoid arthritis}

Joint damage in rheumatoid arthritis is usually assessed radiographically; however, it typically takes $>12$ months for changes to be observable. A Dutch-Canadian team assessed whether four biomarkers, for which assays have recently become available, can predict radiographic damage, in a randomized, prospective, clinical trial in 87 patients with rheumatoid arthritis.

Serum concentrations of the four biomarkers were measured 1, 2, 3 and 4 years after diagnosis. 